Matter sound waves in two-component Bose-Einstein condensates

This article has been downloaded from IOPscience. Please scroll down to see the full text article.

2008 J. Phys. B: At. Mol. Opt. Phys. 41215302

(http://iopscience.iop.org/0953-4075/41/21/215302)

View the table of contents for this issue, or go to the journal homepage for more

Download details:

IP Address: 140.112.50.244

The article was downloaded on 15/10/2010 at 06:16

Please note that terms and conditions apply. 


\title{
Matter sound waves in two-component Bose-Einstein condensates
}

\author{
B B Baizakov ${ }^{1}$, A M Kamchatnov ${ }^{2}$ and M Salerno ${ }^{3}$ \\ ${ }^{1}$ Physical-Technical Institute, Uzbek Academy of Sciences, 100084, Tashkent, Uzbekistan \\ ${ }^{2}$ Institute of Spectroscopy, Russian Academy of Sciences, Troitsk, Moscow Region, 142190, Russia \\ ${ }^{3}$ Dipartimento di Fisica 'E R Caianiello', Consorzio Nazionale Interuniversitario per le Scienze Fisiche \\ della Materia (CNISM), Universitá di Salerno, via S Allende I-84081, Baronissi (SA), Italy \\ E-mail: baizakov@uzsci.net,kamch@isan.troitsk.ru and salerno@sa.infn.it
}

Received 4 August 2008, in final form 3 October 2008

Published 27 October 2008

Online at stacks.iop.org/JPhysB/41/215302

\begin{abstract}
The creation and propagation of sound waves in two-component Bose-Einstein condensates (BECs) are investigated and a new method of wave generation in binary BEC mixtures is proposed. The method is based on a fast change of the inter-species interaction constant and is illustrated for two experimental settings: a drop-like condensate immersed into a second large repulsive condensate, and a binary mixture of two homogeneous repulsive BECs. A mathematical model based on the linearized coupled Gross-Pitaevskii equations is developed and explicit formulae for the space and time dependence of sound waves are provided.

Comparison of the analytical and numerical results shows excellent agreement, confirming the validity of the proposed approach.
\end{abstract}

(Some figures in this article are in colour only in the electronic version)

\section{Introduction}

Wave phenomena in Bose-Einstein condensates (BECs) represent a very active field of research since the first experimental realization of BECs. Matter wave solitons, shock waves and different wave instabilities are among the hot topics of the field presently under investigation (for a recent review see [1]). In particular, dispersive shock waves in BECs consisting of modulated nonlinear periodic waves which decompose into dark soliton trains (e.g., when an initial disturbance is represented by a hole in the density distribution) or eventually into small amplitude waves (e.g., when an initial disturbance is represented by a hump in the density distribution), have been recently investigated. Theory of such waves and soliton trains described by the nonlinear Schrödinger (NLS) equation was developed in [2-4] and applied to their formation in a one-component BEC in [5-7]. Shock waves in discrete NLS models were investigated in [8-10] while experimental observations of shock waves in BECs were reported in [7, 11]. Although these studies were mainly concentrated on single component BECs, binary BEC mixtures, both single species (two distinct spin or hyperfine states of the same atom) [12] and double species [13], have been created and the existence of several kinds of nonlinear excitations in presence of optical lattices has been demonstrated [14]. The dynamics of vector dark solitons in coupled BECs was theoretically investigated in [15] and the possibility of interaction-induced localizations of neutral impurity atoms immersed in homogeneous BECs was considered in [16].

In spite of the intrinsic nonlinearity of BECs and the relevance of nonlinear excitations for applications, small amplitude wave-packets associated with long-wavelength modulations of the condensate (matter sound waves) also appear to be important. These waves naturally arise in the linear or quasi-linear regimes, e.g. when the propagation of small amplitude disturbances on the constant condensate's background is considered. They are generated also in a supersonic flow of BECs past an obstacle where they form so-called 'ship wave' patterns located outside the Mach cone [17-20]. Small amplitude wave-packets should not be confused with dispersive shocks for which larger disturbances or larger nonlinearity are usually required (this is particularly true in the multi-dimensional case). From a mathematical point of view they can be characterized as waves which, opposite to solitons, entirely belong to the continuum spectrum of the linear eigenvalue problem associated with the NLS equation. From a physical point of view, these waves are 
composed of linear modes which have a Bogoliubov-like dispersion relation and which, in a pure quantum description, correspond to elementary excitations of the system. The spectrum of such excitations characterizes the properties of the two-component BEC superfluid [21].

Propagation of sound waves in single-component harmonically trapped BECs was experimentally observed in $[11,22]$ in the form of travelling density perturbations. The effect of optical lattice on the sound propagation in a singlecomponent BEC has been theoretically studied in [23]. Strong dependence of the sound velocity on the intensity of the optical lattice and its dimensionality was revealed. The generation of Cherenkov-like radiation and nucleation of localized structures (dark-antidark solitons in 1D and vortex-lumps in 2D) in two coupled miscible BECs when an obstacle is dragged through them were recently investigated in [24].

Tightly focused far blue-detuned from atomic transitions laser beam applied to the condensate acts as expulsive potential and rapidly pushes atoms from the light field maximum. Depending on the laser power and pulse duration, different kinds of waves are created in the condensate. Smoothly propagating sound waves are created when the laser beam waist is narrow compared to the size of the condensate and the light intensity is small enough. Sound waves in twocomponent BECs were experimentally observed in [25].

While the advantages of experimental techniques for BECs involving laser beams are extremely appealing, there is a crucial drawback when it concerns applications to twocomponent condensates (in [25] the slow light technique was used). The reason for this is connected to the fact that different atomic species feel different light-induced expulsive potentials (due to optical dipole force) and respond to the laser field in different manners. This leads to the appearance of interfering waves which complicate very much the process under investigation. From this point of view the search of alternative methods to generate matter waves in twocomponent BECs is highly desirable.

The aim of this paper is twofold. From one side we investigate, both analytically and numerically, sound waves in two-component BECs generated by the time evolution of a small initial disturbance. On the other side, we present a new method for the generation of different kinds of waves in binary BEC mixtures which is based on the fast change of the inter-species interaction constant. In view of the recent progress made in the manipulation of the intercomponent interactions in binary BEC mixtures [26] by means of Feshbach resonances, the proposed method appears to be experimentally quite feasible and has the advantage that it relies entirely on the internal physical mechanism for the creation of the density disturbance. This method is used to investigate sound waves in two experimental settings: (i) the case of a drop-like condensate immersed into a second large repulsive condensate and (ii) the case of a homogeneous mixture of two repulsive BECs both with finite backgrounds. In case (i) we first create an initial disturbance in the form of a grey or a bright hump (depending on the sign of the interaction) on a finite background by adiabatically turning on the interaction between the drop-like condensate and the surrounding condensate, and then we rapidly remove the interspecies interaction. The energy is then entirely released in the form of sound waves in the background condensate. In case (ii) the initial disturbance is created by a change of the intra- or inter-species interaction constant via optically induced Feshbach resonance. To illustrate our approach, we numerically simulate the creation and evolution of matter sound waves in a two-component BEC. A mathematical model based on the linearized Gross-Pitaevskii equation has been developed and explicit formulae for the space and time dependence of the sound waves dynamics are obtained. Comparison of the analytical and numerical results shows an excellent agreement, confirming the validity of the proposed approach. Such an agreement is not trivial, since the numerical simulations were performed with the nonlinear GP equations and it is well known that even a small nonlinearity can lead to essential consequences after long enough time of evolution. In our case for reasonably long evolution time the deviations from the linear regime remain small and the linear approximation can be successfully applied, a fact that simplifies considerably the analytical description of the phenomena under consideration. Thus, the obtained results can be of interest for the physics of interacting two-component superfluid systems.

The outline of the paper is as follows. In section 2 we illustrate the proposed approach for the one-dimensional setting, by formulating the mathematical model and performing numerical simulations. In this case one component is a bright soliton or drop-like condensate immersed in the second large repulsive condensate with a finite background. Since the first component is on the zero background, after the interspecies interaction is turned off, we consider the waves generated in the second (background) condensate. Analytical formulae describing the spacetime patterns of sound waves in coupled BECs are explicitly derived. In section 3 the proposed method is extended to the two-dimensional case. In section 4 we consider the most interesting and experimentally relevant case of sound waves in two-component repulsive condensates both with a finite background. In this case the inter-species interaction is present during the whole evolution of waves in both condensates, exhibiting interference effects in the dynamics. In section 5 the main results are summarized.

\section{One-dimensional condensates}

\subsection{Decay of a small disturbance of a stationary state to linear wave packets}

Consider a quasi-1D two-component BEC with attractive interaction in one species and repulsive one in the other species. In absence of the interspecies interaction the stationary ground state corresponds to a bright soliton in the attractive condensate and the Thomas-Fermi (TF) distribution in the repulsive one (which can be considered as uniform if the TF radius is much greater than the bright soliton's width). Adiabatic turning on the interspecies interaction leads to the formation of a new stationary state with a hump or a hole in the repulsive $\mathrm{BEC}$, depending on the sign of the interspecies interaction (see figure 1). 

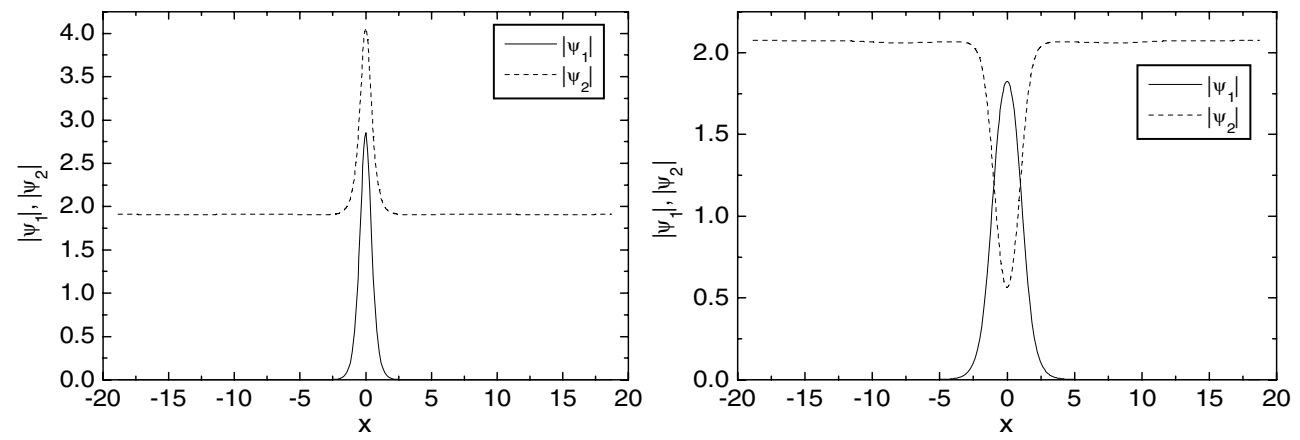

Figure 1. The stationary state of two coupled 1D BECs emerged from the attractive (left panel), and repulsive (right panel) inter-species interactions. Zero background condensate (solid line) is attractive, and finite background condensate (dashed line) is repulsive.

A fast turning the interspecies interaction off will then create a disturbed stationary state in the repulsive condensate. In this section, we shall study evolution of such a perturbation. Since the attractive condensate has a zero background density, we can consider only waves created in the repulsive finite background condensate using the scalar GP equation.

In this case dynamics of the repulsive BEC is governed by the GP equation

$$
\mathrm{i} \psi_{t}+\frac{1}{2} \psi_{x x}+\left(\mu-g|\psi|^{2}\right) \psi=0,
$$

where

$$
\mu=g \rho_{0}=c^{2}
$$

is the chemical potential, $\rho_{0}$ is the density of an undisturbed uniform state, and $c$ denotes the sound velocity of long wavelength waves propagation along such a uniform state. We imply here that the coupling constant $g \equiv g_{1 D}$ corresponds to the 1D geometry of the trap with strong radial confinement $\left(\omega_{\perp} \gg \omega_{x}\right)$ of the BEC.

Let the initial state be described by a disturbed wavefunction

$$
\psi_{0}=\sqrt{\rho_{0}}+\delta \psi_{0}, \quad\left|\delta \psi_{0}\right| \ll \sqrt{\rho_{0}} .
$$

For example, $\delta \psi_{0}$ can describe the hump or the hole created in the density distribution by the interaction with another condensate. Evolution of a small disturbance $\delta \psi$ is governed by a linearized equation (1),

$$
\mathrm{i} \delta \psi_{t}+\frac{1}{2} \delta \psi_{x x}-c^{2}\left(\delta \psi+\delta \psi^{*}\right)=0,
$$

which should be solved with the initial condition

$$
\left.\delta \psi\right|_{t=0}=\delta \psi_{0}(x) .
$$

It is convenient to separate in this equation the real and imaginary parts by introducing

$$
\delta \psi=A+\mathrm{i} B,
$$

so that it transforms to the system

$$
A_{t}+\frac{1}{2} B_{x x}=0, \quad B_{t}-\frac{1}{2} A_{x x}+2 c^{2} A=0 .
$$

The variable $B$ can be easily excluded and we arrive at a single linear equation

$$
A_{t t}-c^{2} A_{x x}+\frac{1}{4} A_{x x x x}=0,
$$

which can be readily solved by the Fourier method. To this end, we note that linear harmonic waves $A \propto \exp [\mathrm{i}(k x-\omega t)]$ satisfy, as it should be, to the Bogoliubov dispersion law,

$$
\omega= \pm \omega(k), \quad \omega(k)=k \sqrt{c^{2}+\frac{k^{2}}{4}} .
$$

Hence the general solution of equation (8) has the form

$$
\begin{gathered}
A(x, t)=\int_{-\infty}^{\infty} W_{1}(k) \mathrm{e}^{\mathrm{i}(k x-\omega(k) t)} \frac{\mathrm{d} k}{2 \pi} \\
+\int_{-\infty}^{\infty} W_{2}(k) \mathrm{e}^{\mathrm{i}(k x+\omega(k) t)} \frac{\mathrm{d} k}{2 \pi},
\end{gathered}
$$

where $W_{1,2}(k)$ are to be determined from the initial conditions:

$$
\begin{aligned}
& A(x, 0) \equiv A_{0}(x)=\int_{-\infty}^{\infty}\left[W_{1}(k)+W_{2}(k)\right] \mathrm{e}^{\mathrm{i} k x} \frac{\mathrm{d} k}{2 \pi} \\
& A_{t}(x, 0) \equiv A_{t 0}(x)=-\mathrm{i} \int_{-\infty}^{\infty} \omega(k)\left[W_{1}(k)-W_{2}(k)\right] \mathrm{e}^{\mathrm{i} k x} \frac{\mathrm{d} k}{2 \pi}
\end{aligned}
$$

The inverse Fourier transform gives the equations

$A_{0}(k)=W_{1}(k)+W_{2}(k), \quad A_{t 0}(k)=-\mathrm{i} \omega(k)\left[W_{1}(k)-W_{2}(k)\right]$, which readily provide

$$
\begin{aligned}
& W_{1}(k)=\frac{1}{2}\left(A_{0}(k)+\frac{\mathrm{i}}{\omega(k)} A_{t 0}(k)\right), \\
& W_{2}(k)=\frac{1}{2}\left(A_{0}(k)-\frac{\mathrm{i}}{\omega(k)} A_{t 0}(k)\right) .
\end{aligned}
$$

The first equation (7) allows us to express $A_{t 0}(k)$ in terms of $B_{0}(k), A_{t 0}(k)=\left(k^{2} / 2\right) B_{0}(k)$. At last, $A(k)$ and $B(k)$ can be related with disturbances of the density and the phase in BEC, $\psi=\sqrt{\rho_{0}+\delta \rho} \exp (\mathrm{i} \delta \phi) \cong \sqrt{\rho_{0}}+\frac{\delta \rho}{2 \sqrt{\rho_{0}}}+\mathrm{i} \sqrt{\rho_{0}} \delta \phi$, so that

$$
A=\frac{\delta \rho}{2 \sqrt{\rho_{0}}}, \quad B=\sqrt{\rho_{0}} \delta \phi .
$$

Hence, equations (12) take the form

$$
\begin{aligned}
& W_{1}(k)=\frac{1}{4 \sqrt{\rho_{0}}}\left(\delta \rho_{0}(k)+\mathrm{i} \frac{k^{2} \rho_{0}}{\omega(k)} \delta \phi_{0}(k)\right), \\
& W_{2}(k)=\frac{1}{4 \sqrt{\rho_{0}}}\left(\delta \rho_{0}(k)-\mathrm{i} \frac{k^{2} \rho_{0}}{\omega(k)} \delta \phi_{0}(k)\right) .
\end{aligned}
$$



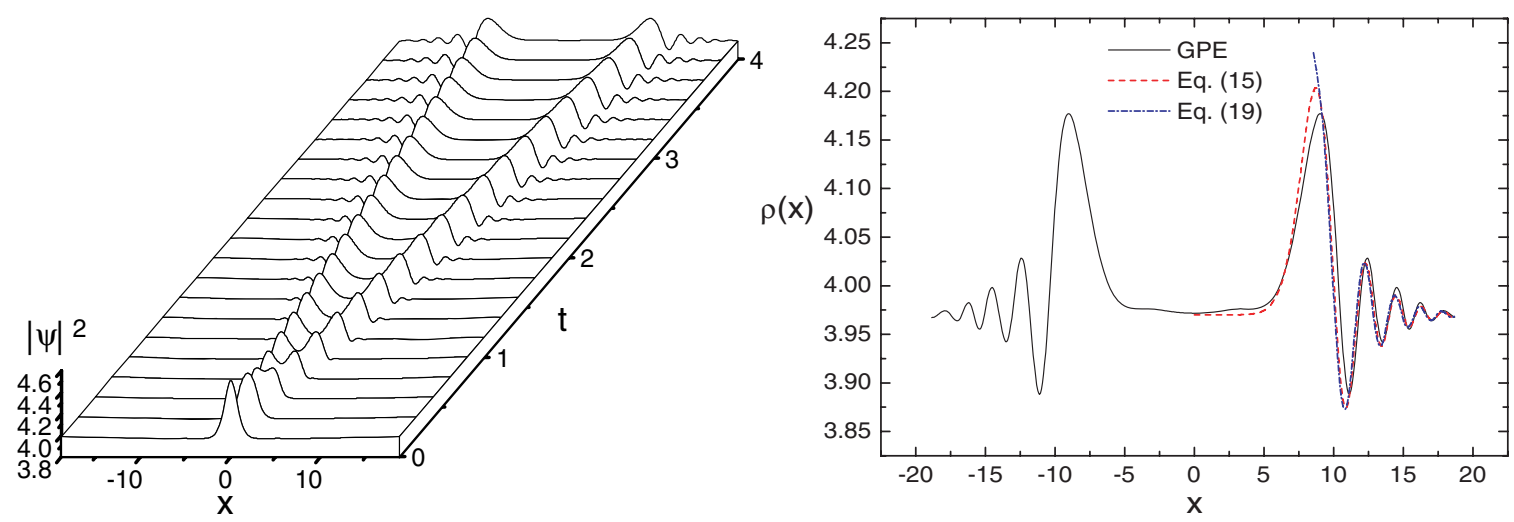

Figure 2. Left panel: time evolution of the density disturbance in the background BEC $\left(\rho(x)=|\psi(x)|^{2}\right)$, when the inter-species interaction constant is set to zero $\left(g_{12}=0\right)$ at $t=0$, according to the GP equation (37) with $g_{22}=g=1$. Right panel: comparison of the exact analytic solution (15), asymptotic approximation (19), and numerical solution of the GP equation (37) for the density disturbance, corresponding to $t=4$ and $c=2$. The initial disturbance has a gaussian form (21) with $a=0.95$. Space dependence for analytical solutions is shown for $x>0$ at the moment $t=4$. A similar calculation gives a symmetric distribution for $x<0$.

Let us consider, for the sake of definiteness, the case of an initial disturbance of density only, $\delta \phi_{0}=0$, so that $W_{1}(k)=W_{2}(k)=\delta \rho_{0}(k) /\left(4 \sqrt{\rho_{0}}\right)$ and then

$$
\begin{aligned}
\delta \rho(x, t) & =2 \sqrt{\rho_{0}} A(x, t) \\
& =\frac{1}{4 \pi} \int_{-\infty}^{\infty} \delta \rho_{0}(k)\left[\mathrm{e}^{\mathrm{i}(k x-\omega(k) t)}+\mathrm{e}^{\mathrm{i}(k x+\omega(k) t)}\right] \mathrm{d} k \\
& =\frac{1}{\pi} \int_{0}^{\infty} \delta \rho_{0}(k) \cos (k x) \cos \left(t k \sqrt{c^{2}+\frac{k^{2}}{4}}\right) \mathrm{d} k .
\end{aligned}
$$

The integral here can be estimated for large $t$, as usual, by the method of the stationary phase. For $x>0$, only the first exponent gives a contribution to the integral,

$$
\delta \rho(x, t)=\frac{1}{4 \pi} \int_{-\infty}^{\infty} \delta \rho_{0}(k) \mathrm{e}^{\mathrm{i} t f(k)} \mathrm{d} k, \quad x>0,
$$

where

$$
f(k)=k\left(\frac{x}{t}-\sqrt{c^{2}+\frac{k^{2}}{4}}\right) .
$$

The values of $k$ at the points of the stationary phase determined by the condition $\mathrm{d} f / \mathrm{d} k=0$ are equal to $(x>c t)$

$k= \pm k_{0}(x, t)$

$k_{0}(x, t)=\frac{c}{\sqrt{2}}\left[\left(\frac{x}{c t}\right)^{2}-4+\frac{x}{c t} \sqrt{\left(\frac{x}{c t}\right)^{2}+8}\right]^{1 / 2}$,

and integration yields

$$
\delta \rho(x, t)=\frac{\delta \rho_{0}\left(k_{0}\right)}{\sqrt{2 \pi t\left|\frac{\mathrm{d}^{2} f}{\mathrm{~d} k^{2}}\right|_{0}}} \cos \left[t f\left(k_{0}\right)-\frac{\pi}{4}\right],
$$

where

$$
\left|\frac{\mathrm{d}^{2} f}{\mathrm{~d} k^{2}}\right|_{0}=\frac{k_{0}\left(6 c^{2}+k_{0}^{2}\right)}{\left(4 c^{2}+k_{0}^{2}\right)^{3 / 2}} .
$$

Here $k_{0}$ is a function of $x$ and $t$ determined by equation (18). In figure 2 we compare the exact solution (15) corresponding to a gaussian initial disturbance

$$
\begin{array}{r}
\delta \rho_{0}(x)=\frac{1}{\sqrt{\pi} a} \exp \left(-\frac{x^{2}}{a^{2}}\right), \\
\delta \rho_{0}(k)=\exp \left(-\frac{k^{2} a^{2}}{4}\right),
\end{array}
$$

with its approximation (19), and corroborate these two curves with numerical solution of the GP equation where we introduce as initial condition the wave profiles shown in the left panel of figure 1. It is remarkable that the asymptotic formula (19) is quite accurate even for not very large values of $t$ along almost all wave packet where $|x|>c t$ (see figure 2).

It is worth mentioning that there is a significant difference between two phenomena which at first sight look very similar-namely, between linear wave packets discussed here and dispersive shocks. In both cases wave trains are generated from an initial disturbance and they can be represented as modulated periodic waves. However, in case of wave packets the evolution is a purely dispersive phenomenon, whereas for dispersive shocks the role of nonlinearity is crucial. Hence, as is well known, a linear wave packet propagates as a whole with the group velocity $v_{g}=\mathrm{d} \omega / \mathrm{d} k$ estimated for some characteristic value of the wave vector $k$, and this velocity is always greater that the sound velocity. This is evident both from the dispersion relation (9) and the results of our numerical simulations. By contrast, a dispersive shock can be represented as a 'soliton lattice' which describes a transition between regions with different wave parameters, e.g., a jump of the condensate density appearing after wave breaking of a large initial disturbance (see, e.g., [6, 7]). Asymptotically for a sufficiently long evolution time such a 'lattice' can evolve into a train of solitons well separated from each other [4], which always propagate with velocities less than the sound velocity. This also follows from the dispersion relation (9) when it is considered that the soliton tails are described by a linear theory with replacement of a harmonic wave $\exp [\mathrm{i}(k x-\omega t)]$ by an exponential decay law $\exp [ \pm \kappa(x-V t)]$, where $\kappa$ is an inverse half-width of the soliton and $V$ is its velocity. Hence, the 
velocity $V$ is associated with $\kappa$ through the expression obtained from (9) by the replacements $\mathrm{i} k \rightarrow \pm \kappa, \mathrm{i} \omega \rightarrow \pm \kappa V$. As a result we get $V=\sqrt{c^{2}-\kappa^{2} / 4}$, which yields $V<c$. This is a quite general statement which is also true for other linear wave packets considered below and it stands in sharp contrast with dispersive shock waves which, although displaying similar profiles at short times, have velocities smaller than the sound velocity and asymptotically separate into trains of solitons.

All numerical simulations are performed by a split-step fast Fourier transform (FFT) [27] on a spatial domain of length $L=32 \pi$ with 2048 modes in the $1 \mathrm{D}$ case, and $512 \times 512$ modes in the $2 \mathrm{D}$ case (see below). The time step was $\delta t=0.001$. To control the accuracy of numerical results we monitored the norms (number of atoms) of the BEC components which showed conservation during the time evolution to precision better than $10^{-3}$ in normalized units. Our numerical procedure represents a straightforward extension of the split-step FFT technique that is used for the single-component nonlinear Schrödinger equation [28]. It is appropriate to mention that the significance of numerical methods for investigation of multi-component and multidimensional BECs has motivated the development of highly effective techniques, as reviewed in [29]. Among the powerful numerical methods for finding the ground states and timeevolution of vector GP equations, a time-splitting sine-spectral method [30] can be particularly mentioned. The merits of this method are that it is explicit, unconditionally stable, time reversible and is of spectral-order accuracy in space and second-order accuracy in time.

\section{Two-dimensional condensates}

\subsection{Decay of small disturbances to wave packets}

This problem can be solved by the same method which was used in $1 \mathrm{D}$ case with replacement $\partial^{2} / \partial x^{2} \rightarrow \Delta_{\perp} \equiv$ $\partial^{2} / \partial x^{2}+\partial^{2} / \partial y^{2}$. As a result we obtain the general solution in the form

$$
\begin{gathered}
A(\mathbf{r}, t)=\int W_{1}(\mathbf{k}) \mathrm{e}^{\mathrm{i}(\mathbf{k r}-\omega(k) t)} \frac{\mathrm{d} \mathbf{k}}{(2 \pi)^{2}} \\
+\int W_{2}(\mathbf{k}) \mathrm{e}^{\mathrm{i}(\mathbf{k r}+\omega(k) t)} \frac{\mathrm{d} \mathbf{k}}{(2 \pi)^{2}},
\end{gathered}
$$

where $W_{1,2}(\mathbf{k})$ are determined again from the initial conditions, which gives

$$
\begin{aligned}
& W_{1}(\mathbf{k})=\frac{1}{4 \sqrt{\rho_{0}}}\left(\delta \rho_{0}(\mathbf{k})+\mathrm{i} \frac{k^{2} \rho_{0}}{\omega(k)} \delta \phi_{0}(\mathbf{k})\right) \\
& W_{2}(\mathbf{k})=\frac{1}{4 \sqrt{\rho_{0}}}\left(\delta \rho_{0}(\mathbf{k})-\mathrm{i} \frac{k^{2} \rho_{0}}{\omega(k)} \delta \phi_{0}(\mathbf{k})\right) .
\end{aligned}
$$

For cylindrically symmetric initial disturbance of density only $\left(\delta \phi_{0}(\mathbf{k})=0\right)$ we get for the wave of density $\delta \rho(r, t)=$ $2 \sqrt{\rho_{0}} A(r, t)$ the expressions similar to equations (15),

$$
\begin{aligned}
& \delta \rho(r, t)=\frac{1}{8 \pi^{2}} \int_{0}^{2 \pi} \int_{0}^{\infty} \delta \rho_{0}(k)\left[\mathrm{e}^{\mathrm{i}(k r \cos \varphi-\omega(k) t)}\right. \\
& \left.+\mathrm{e}^{\mathrm{i}(k r \cos \varphi+\omega(k) t)}\right] k \mathrm{~d} k \mathrm{~d} \varphi \\
& =\frac{1}{2 \pi} \int_{0}^{\infty} \delta \rho_{0}(k) \cos \left(t k \sqrt{c^{2}+\frac{k^{2}}{4}}\right) J_{0}(k r) k \mathrm{~d} k,
\end{aligned}
$$

where

$$
J_{0}(z)=\frac{1}{2 \pi} \int_{0}^{\infty} \mathrm{e}^{\mathrm{i} z \cos \varphi} \mathrm{d} \varphi
$$

is the Bessel function of the first kind.

For $k r \gg 1$ the integral in (24) can be estimated by the method of the stationary phase with the use of the asymptotic expression for the Bessel function,

$$
J_{0}(k r) \approx \sqrt{\frac{2}{\pi k r}} \cos (k r-\pi / 4) .
$$

As a result we get

$$
\delta \rho(r, t)=\frac{\delta \rho_{0}\left(k_{0}\right) \sqrt{k_{0}}}{2 \pi \sqrt{r t\left|\frac{\mathrm{d}^{2} f}{\mathrm{~d} k^{2}}\right|_{0}}} \sin \left[t f\left(k_{0}\right)\right],
$$

where

$$
\begin{aligned}
& k_{0}(r, t)=\frac{c}{\sqrt{2}}\left[\left(\frac{r}{c t}\right)^{2}-4+\frac{r}{c t} \sqrt{\left(\frac{r}{c t}\right)^{2}+8}\right]^{1 / 2}, \quad r>c t \\
& f(k)=k\left(\frac{r}{t}-\sqrt{c^{2}+\frac{k^{2}}{4}}\right)
\end{aligned}
$$

and $\left|\mathrm{d}^{2} f / \mathrm{d} k^{2}\right|_{0}$ is given by (20).

In order to compare the analytical predictions with numerical solution of the GP equation we need to perform a sequence of simulations as in the 1D case. However, there is an essential difference in the $2 \mathrm{D}$ case due to the fact that no stable localized solution for the drop-like condensate exists for the 2D GP equation without an external potential. In this regard we recall that the attractive 2D NLS equation admits a localized solution, the so-called Townes soliton, which exists only for a critical value of the norm (number of atoms) and is unstable against small fluctuations of the critical norm (overcritical norms lead to collapse, while under-critical ones lead to background decay). To avoid this problem, we prepare the drop-like condensate in the ground state of the parabolic trap $V(x, y)=v_{0}\left(x^{2}+y^{2}\right)$ with strength $v_{0}=1$, using the imaginary-time relaxation method [31]. Then we introduce the obtained ground state solution for the drop-like condensate $\psi_{1}(x, y)$ and constant amplitude solution for the background condensate $\left(\psi_{2}(x, y)=2\right)$ as initial condition in the coupled GP equation with $g_{12}=0$, and slowly increase the interspecies interaction constant to a finite value $g_{12}=-0.5$ (see figure 3, left panel). It is remarkable that after the localized coupled state has been created by this method, it remains stable even after the parabolic potential is removed, due to the stabilizing effect induced by the interspecies coupling (this state exists only due to coupling). As a result the initial state shown in the right panel of figure 3 is obtained. The density waves in the background condensate can be generated by fast turning off the interaction constant $g_{12} \rightarrow 0$. The decaying density hump induces a circular wave in the background BEC travelling outward from the centre. We have shown in figure 4 the dependence of $\delta \rho$ on $r$ at fixed value of time $(t=4)$ for the Gaussian initial distribution

$\delta \rho_{0}(r)=\frac{1}{\pi a^{2}} \exp \left(-\frac{r^{2}}{a^{2}}\right), \quad \delta \rho_{0}(k)=\exp \left(-\frac{k^{2} a^{2}}{4}\right)$, 

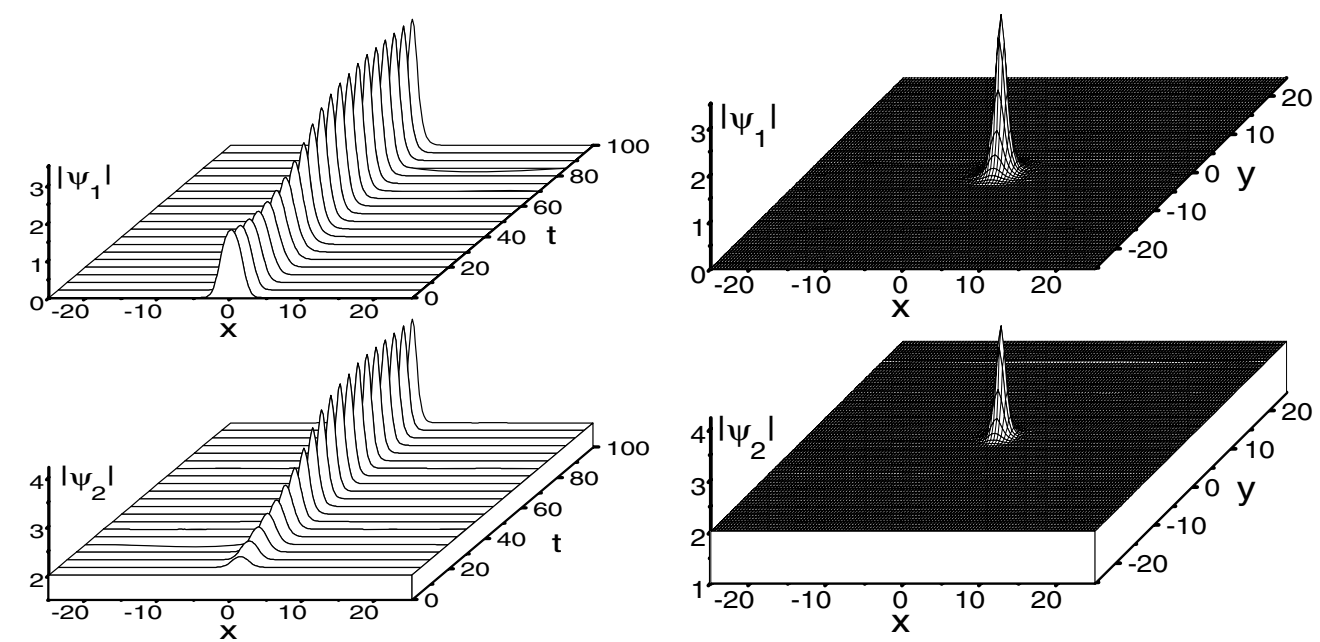

Figure 3. Left panel: formation of a density hump on the background condensate $\psi_{2}(x, y)$, shown at the $y=0$ cross section, when the inter-species interaction constant is slowly raised from zero according to $g_{12}=-0.5 \tanh \left(5 t / t_{\text {end }}\right)$, with $t_{\text {end }}=100$. Due to inter-species attraction, the drop-like repulsive condensate $\psi_{1}(x, y)$ with a norm $N=6 \pi$ becomes more narrow. Right panel: the final stationary coupled state of the two repulsive BECs with $g_{11}=1, g_{22}=1$, with attractive inter-species interaction constant $g_{12}=-0.5$.
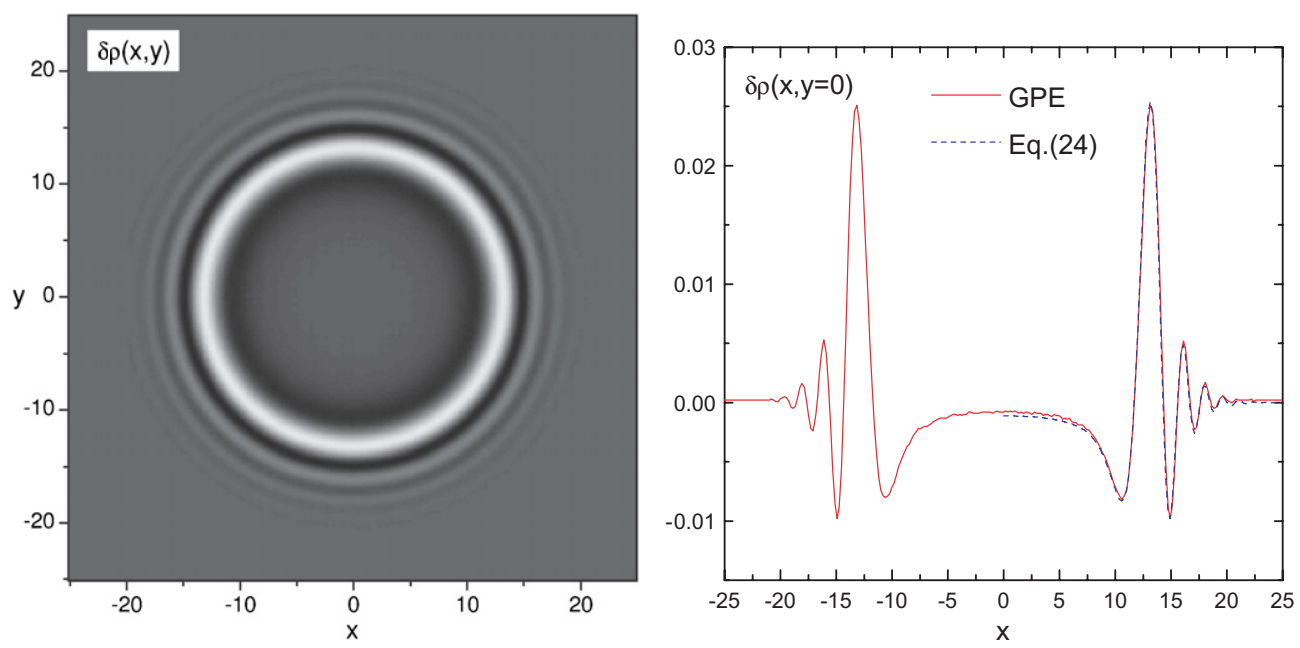

Figure 4. Left panel: density plot $\rho(r)=|\psi(x, y)|^{2}$ of the repulsive background condensate at $t=4$ after turning off the inter-species interaction constant $g_{12}=0$, according to numerical solution of the GP equation. White stripes correspond to higher density. Right panel: comparison between analytical expression (24), for the density disturbance in the background condensate at $t=4$ with corresponding numerical solution of the GP equation $\left(|\psi(x, y=0)|^{2}-\rho_{0}\right)$, shown for $y=0$ cross section. Parameters: $\rho_{0}=9, c=3, a=1$.

with $a=1$ calculated according to the exact expression (24). The asymptotic approximation (27) agrees very well with the exact expression (24) at $x>c t$ (not shown in figure because the corresponding lines overlap).

\section{Two-component condensates}

Let us now consider the relevant case of a homogeneous binary BEC mixture, when both components have a finite background. Two-component condensates are described in our non-dimensional variables by the equations

$\mathrm{i} \frac{\partial \psi_{1}}{\partial t}+\frac{1}{2} \Delta \psi_{1}+\left[\mu_{1}-\left(g_{11}\left|\psi_{1}\right|^{2}+g_{12}\left|\psi_{2}\right|^{2}\right)\right] \psi_{1}=0$,

$\mathrm{i} \frac{\partial \psi_{2}}{\partial t}+\frac{1}{2} \Delta \psi_{2}+\left[\mu_{2}-\left(g_{12}\left|\psi_{1}\right|^{2}+g_{22}\left|\psi_{2}\right|^{2}\right)\right] \psi_{2}=0$, where

$$
\mu_{1}=g_{11} \rho_{10}+g_{12} \rho_{20}, \quad \mu_{2}=g_{12} \rho_{10}+g_{22} \rho_{20}
$$

are chemical potentials of two species with equilibrium uniform densities $\rho_{10}$ and $\rho_{20}$; we keep here the general notation for the Laplacian $\Delta$ which will be specified later depending on the number of spatial dimensions in the problem under consideration.

In disturbed condensates we have $\psi_{1}=\sqrt{\rho_{10}}+\delta \psi_{1}, \psi_{2}=$ $\sqrt{\rho_{20}}+\delta \psi_{2}$, where evolution of disturbances is governed by the linearized equations

$$
\begin{aligned}
\mathrm{i} \delta \psi_{1} & +\frac{1}{2} \Delta \delta \psi_{1}-g_{11} \rho_{10}\left(\delta \psi_{1}+\delta \psi_{1}^{*}\right) \\
& -g_{12} \sqrt{\rho_{10} \rho_{20}}\left(\delta \psi_{2}+\delta \psi_{2}^{*}\right)=0 \\
\mathrm{i} \delta \psi_{2} & +\frac{1}{2} \Delta \delta \psi_{2}-g_{12} \sqrt{\rho_{10} \rho_{20}}\left(\delta \psi_{1}+\delta \psi_{1}^{*}\right) \\
& -g_{22} \rho_{20}\left(\delta \psi_{2}+\delta \psi_{2}^{*}\right)=0 .
\end{aligned}
$$


As before, we introduce

$$
\delta \psi_{1}=A_{1}+\mathrm{i} B_{1}, \quad \delta \psi_{2}=A_{2}+\mathrm{i} B_{2},
$$

so that after substitution into (33), separation of real and imaginary parts followed by exclusion of $B_{1}$ and $B_{2}$ we arrive at the system

$$
\begin{aligned}
& A_{1, t t}-g_{11} \rho_{10} \Delta A_{1}+\frac{1}{4} \Delta^{2} A_{1}-g_{12} \sqrt{\rho_{10} \rho_{20}} \Delta A_{2}=0, \\
& A_{2, t t}-g_{22} \rho_{20} \Delta A_{2}+\frac{1}{4} \Delta^{2} A_{2}-g_{12} \sqrt{\rho_{10} \rho_{20}} \Delta A_{1}=0 .
\end{aligned}
$$

For plane waves $A_{1}, A_{2} \propto \exp [\mathrm{i}(\mathbf{k r}-\omega t)]$ we reproduce the known dispersion laws

$$
\omega_{ \pm}=k \sqrt{c_{ \pm}^{2}+\frac{k^{2}}{4}}
$$

where the sound velocities corresponding to infinite wavelengths are equal to

$$
\begin{aligned}
c_{ \pm} & =\frac{1}{\sqrt{2}}\left[g_{11} \rho_{10}+g_{22} \rho_{20}\right. \\
& \left. \pm \sqrt{\left(g_{11} \rho_{10}-g_{22} \rho_{20}\right)^{2}+4 g_{12}^{2} \rho_{10} \rho_{20}}\right]^{1 / 2} .
\end{aligned}
$$

The ratios $A_{1} / A_{2}$ in these two modes of linear waves are given by

$$
\frac{A_{1}}{A_{2}}=\frac{2 g_{12} \sqrt{\rho_{10} \rho_{20}}}{g_{22} \rho_{20}-g_{11} \rho_{10} \pm\left(c_{+}^{2}-c_{-}^{2}\right)} .
$$

Thus, the general solution of the system (35) is given by

$$
\begin{gathered}
A_{1}(\mathbf{r}, t)=\int W_{11}^{+}(\mathbf{k}) \mathrm{e}^{\mathrm{i}\left(\mathbf{k r}-\omega_{+} t\right)} \frac{\mathrm{d}^{D} k}{(2 \pi)^{D}} \\
+\int W_{12}^{+}(\mathbf{k}) \mathrm{e}^{\mathrm{i}\left(\mathbf{k r}+\omega_{+} t\right)} \frac{\mathrm{d}^{D} k}{(2 \pi)^{D}} \\
+\int W_{11}^{-}(\mathbf{k}) \mathrm{e}^{\mathrm{i}\left(\mathbf{k r}-\omega_{-} t\right)} \frac{\mathrm{d}^{D} k}{(2 \pi)^{D}} \\
+\int W_{12}^{-}(\mathbf{k}) \mathrm{e}^{\mathrm{i}\left(\mathbf{k r}+\omega_{-} t\right)} \frac{\mathrm{d}^{D} k}{(2 \pi)^{D}}, \\
A_{2}(\mathbf{r}, t)=\int W_{21}^{+}(\mathbf{k}) \mathrm{e}^{\mathrm{i}\left(\mathbf{k r}-\omega_{+} t\right)} \frac{\mathrm{d}^{D} k}{(2 \pi)^{D}} \\
+\int W_{22}^{+}(\mathbf{k}) \mathrm{e}^{\mathrm{i}\left(\mathbf{k r}+\omega_{+} t\right)} \frac{\mathrm{d}^{D} k}{(2 \pi)^{D}} \\
+\int W_{21}^{-}(\mathbf{k}) \mathrm{e}^{\mathrm{i}\left(\mathbf{k r}-\omega_{-} t\right)} \frac{\mathrm{d}^{D} k}{(2 \pi)^{D}} \\
+\int W_{22}^{-}(\mathbf{k}) \mathrm{e}^{\mathrm{i}\left(\mathbf{k r}+\omega_{-} t\right)} \frac{\mathrm{d}^{D} k}{(2 \pi)^{D}},
\end{gathered}
$$

where $D$ denotes dimension of the space. According to (38) the Fourier components are related with each other by the equations

$$
\frac{W_{11}^{ \pm}}{W_{21}^{ \pm}}=\frac{W_{12}^{ \pm}}{W_{22}^{ \pm}}=\frac{2 g_{12} \sqrt{\rho_{10} \rho_{20}}}{g_{22} \rho_{20}-g_{11} \rho_{10} \pm\left(c_{+}^{2}-c_{-}^{2}\right)},
$$

that is only four of them are independent of each other and should be determined from the initial conditions.
To simplify the notation, in what follows we shall consider the case when there exist only initial disturbances $\delta \rho_{10}(\mathbf{r}), \delta \rho_{20}(\mathbf{r})$ of the densities. Then

$$
W_{11}^{ \pm}=W_{12}^{ \pm} \equiv W_{1}^{ \pm}, \quad W_{21}^{ \pm}=W_{22}^{ \pm} \equiv W_{2}^{ \pm},
$$

and these four functions must satisfy the linear system

$$
\begin{gathered}
A_{10}(\mathbf{k})=2\left(W_{1}^{+}+W_{1}^{-}\right), \\
A_{20}(\mathbf{k})=2\left(W_{2}^{+}+W_{2}^{-}\right), \\
\frac{W_{1}^{+}}{W_{2}^{+}}=\frac{2 g_{12} \sqrt{\rho_{10} \rho_{20}}}{g_{22} \rho_{20}-g_{11} \rho_{10}+\left(c_{+}^{2}-c_{-}^{2}\right)}, \\
\frac{W_{1}^{-}}{W_{2}^{-}}=\frac{2 g_{12} \sqrt{\rho_{10} \rho_{20}}}{g_{22} \rho_{20}-g_{11} \rho_{10}-\left(c_{+}^{2}-c_{-}^{2}\right)},
\end{gathered}
$$

which can be readily solved. Taking into account that

$$
A_{10}(\mathbf{k})=\frac{1}{2 \sqrt{\rho_{10}}} \delta \rho_{10}(\mathbf{k}), \quad A_{20}(\mathbf{k})=\frac{1}{2 \sqrt{\rho_{20}}} \delta \rho_{20}(\mathbf{k}),
$$

we can find the waves of the densities of the two species.

In $1 \mathrm{D}$ case the final formulae read

$\delta \rho_{1}(x, t)=\frac{1}{2 \pi} \int_{0}^{\infty} \delta \rho_{1}(k) \cos k x$

$$
\begin{aligned}
& \times\left[\left(1+\frac{g_{11} \rho_{10}-g_{22} \rho_{20}}{c_{+}^{2}-c_{-}^{2}}\right) \cos \left(t k \sqrt{c_{+}^{2}+\frac{k^{2}}{4}}\right)\right. \\
& \left.+\left(1-\frac{g_{11} \rho_{10}-g_{22} \rho_{20}}{c_{+}^{2}-c_{-}^{2}}\right) \cos \left(t k \sqrt{c_{-}^{2}+\frac{k^{2}}{4}}\right)\right] \mathrm{d} k \\
& +\frac{g_{12} \rho_{10}}{\pi\left(c_{+}^{2}-c_{-}^{2}\right)} \int_{0}^{\infty} \delta \rho_{2}(k) \cos k x\left[\cos \left(t k \sqrt{c_{+}^{2}+\frac{k^{2}}{4}}\right)\right. \\
& \left.-\cos \left(t k \sqrt{c_{-}^{2}+\frac{k^{2}}{4}}\right)\right] \mathrm{d} k,
\end{aligned}
$$

$$
\begin{aligned}
\delta \rho_{2}(x, t) & =\frac{1}{2 \pi} \int_{0}^{\infty} \delta \rho_{2}(k) \cos k x \\
\times & {\left[\left(1-\frac{g_{11} \rho_{10}-g_{22} \rho_{20}}{c_{+}^{2}-c_{-}^{2}}\right) \cos \left(t k \sqrt{c_{+}^{2}+\frac{k^{2}}{4}}\right)\right.} \\
+ & \left.\left(1+\frac{g_{11} \rho_{10}-g_{22} \rho_{20}}{c_{+}^{2}-c_{-}^{2}}\right) \cos \left(t k \sqrt{c_{-}^{2}+\frac{k^{2}}{4}}\right)\right] \mathrm{d} k \\
+ & \frac{g_{12} \rho_{20}}{\pi\left(c_{+}^{2}-c_{-}^{2}\right)} \int_{0}^{\infty} \delta \rho_{1}(k) \cos k x\left[\cos \left(t k \sqrt{c_{+}^{2}+\frac{k^{2}}{4}}\right)\right. \\
& \left.-\cos \left(t k \sqrt{c_{-}^{2}+\frac{k^{2}}{4}}\right)\right] \mathrm{d} k .
\end{aligned}
$$

Similar formulae are obtained for two-dimensional cylindrically symmetric waves as

$$
\begin{aligned}
\delta \rho_{1}(r, t) & =\frac{1}{4 \pi} \int_{0}^{\infty} \delta \rho_{1}(k) J_{0}(k r) \\
\times & {\left[\left(1+\frac{g_{11} \rho_{10}-g_{22} \rho_{20}}{c_{+}^{2}-c_{-}^{2}}\right) \cos \left(t k \sqrt{c_{+}^{2}+\frac{k^{2}}{4}}\right)\right.} \\
+ & \left.\left(1-\frac{g_{11} \rho_{10}-g_{22} \rho_{20}}{c_{+}^{2}-c_{-}^{2}}\right) \cos \left(t k \sqrt{c_{-}^{2}+\frac{k^{2}}{4}}\right)\right] k \mathrm{~d} k
\end{aligned}
$$




$$
\begin{aligned}
&+ \frac{g_{12} \rho_{10}}{2 \pi\left(c_{+}^{2}-c_{-}^{2}\right)} \int_{0}^{\infty} \delta \rho_{2}(k) J_{0}(k r) \\
& \times {\left[\cos \left(t k \sqrt{c_{+}^{2}+\frac{k^{2}}{4}}\right)-\cos \left(t k \sqrt{c_{-}^{2}+\frac{k^{2}}{4}}\right)\right] k \mathrm{~d} k } \\
& \delta \rho_{2}(r, t)=\frac{1}{4 \pi} \int_{0}^{\infty} \delta \rho_{2}(k) J_{0}(k r) \\
& \times\left[\left(1-\frac{g_{11} \rho_{10}-g_{22} \rho_{20}}{c_{+}^{2}-c_{-}^{2}}\right) \cos \left(t k \sqrt{c_{+}^{2}+\frac{k^{2}}{4}}\right)\right. \\
&\left.+\left(1+\frac{g_{11} \rho_{10}-g_{22} \rho_{20}}{c_{+}^{2}-c_{-}^{2}}\right) \cos \left(t k \sqrt{c_{-}^{2}+\frac{k^{2}}{4}}\right)\right] k \mathrm{~d} k \\
&+\frac{g_{12} \rho_{10}}{2 \pi\left(c_{+}^{2}-c_{-}^{2}\right)} \int_{0}^{\infty} \delta \rho_{1}(k) J_{0}(k r)\left[\cos \left(t k \sqrt{c_{+}^{2}+\frac{k^{2}}{4}}\right)\right. \\
&\left.-\cos \left(t k \sqrt{c_{-}^{2}+\frac{k^{2}}{4}}\right)\right] k \mathrm{~d} k .
\end{aligned}
$$

It is easy to see that if there is no interaction between two components of BECs, then these formulae reduce to the already known results for one-component condensates.

Integrals in (45)-(48) can be estimated by the method of stationary phase which gives the approximate expressions for disturbances of the densities in the regions $x>c_{+} t$ or $r>c_{+} t$ where we can observe the interference pattern of two modes.

The present setting with homogeneously mixed two repulsive BECs corresponds to the most relevant experimental situation. The case of two repulsive 1D BECs is akin to the previously discussed one (see figure 1), if there is a dark soliton in one component. When the repulsive inter-species interaction is slowly turned on, a density hump emerges in the other component, as illustrated in figure 5. Linear waves can then be generated by a fast turning off of the inter-species interaction.

Another approach to generation of density disturbances in coupled repulsive BECs involves local change of the intraor inter-species interaction constants by a tightly focused laser beam via optically induced Feshbach resonance [32, 33]. In this regard, we recall that a Feshbach resonance occurs when a colliding pair of atoms is resonantly coupled to a molecular bound state. A magnetically tunable Feshbach resonance is based on Zeeman shifting a bound molecular state into resonance with the scattering state [34]. Alternative coupling schemes for inducing Feshbach resonance have been proposed [32], which uses optical coupling of the scattering state with the molecular state. This scheme, often referred to as optically induced Feshbach resonance, can be controlled via laser detuning and laser power. An experimental demonstration of changing the atomic scattering length over one order of magnitude (from $10 a_{0}$ to $190 a_{0}$, where $a_{0}$ is Bohr radius) was demonstrated with ${ }^{87} \mathrm{Rb}$ atoms using a moderate laser intensity of about $500 \mathrm{~W} \mathrm{~cm}^{-2}$ [33].

In the context of a new approach to generation of sound waves in coupled BECs developed in this work, the optically
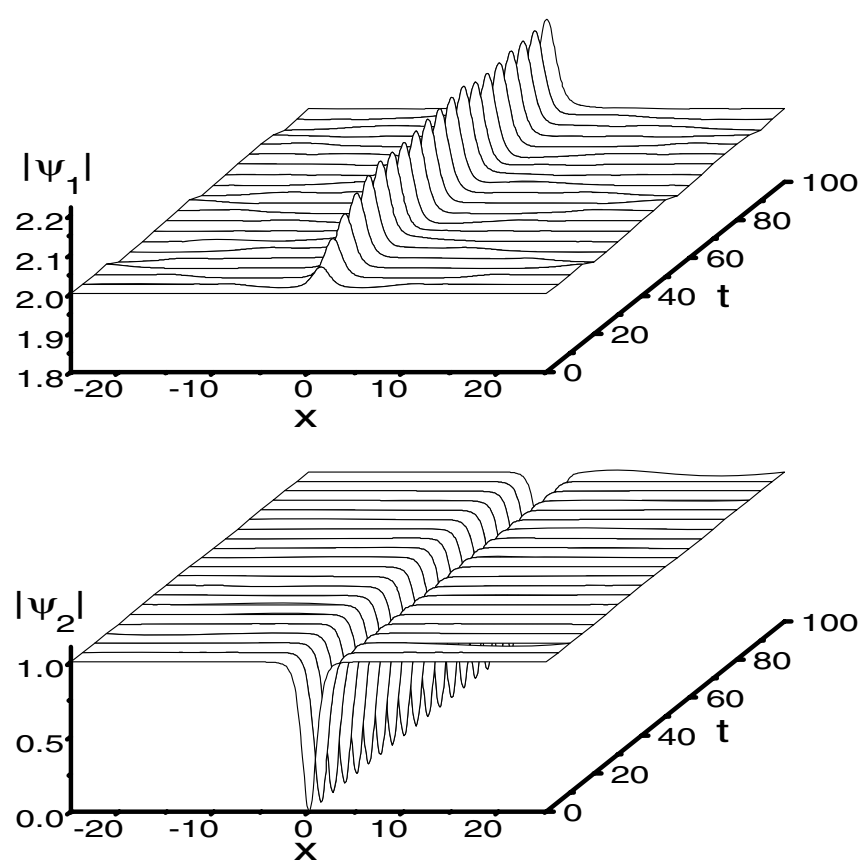

Figure 5. Formation of a density defect in two coupled $1 D$ repulsive BECs with initial wave amplitudes $\psi_{1}(x)=2, \psi_{2}(x)=\tanh (x)$, and $g_{11}=1, g_{22}=1$, when the repulsive inter-species interaction is slowly turned on according to law $g_{12}=0.5 \tanh \left(5 t / t_{\text {end }}\right)$, with $t_{\text {end }}=100$. A dark soliton in one component gives rise to a density hump in the other component.

induced Feshbach resonance has important advantages over its usual magnetic counterpart. In particular, by the optical method complex spatial intensity distributions can be easily produced which result in corresponding scattering length pattern across the sample. In addition, the intensity and detuning of optical fields can be varied much more rapidly than those of magnetic fields.

Stationary density perturbations in the form of coupled localized states on finite backgrounds can emerge in mixture BECs when the intra- or inter-species interaction constants are locally changed as illustrated in figure 6 . Note that these are not self-trapped states like vector solitons of coupled GP equations [35-37], they decay out when the local change of the interaction coefficient is removed. The experimental observation of long-lived dark-bright solitons, with lifetimes of up to several seconds, and their dynamics in a two-component BEC (two hyperfine states of ${ }^{87} \mathrm{Rb}$ ) has been recently reported in [38].

After the density defect (hump or hole) has been created, a fast change of the inter-species interaction coefficient leads to generation of waves in the condensate caused by decaying density disturbance. These results are illustrated in figure 7 , where comparison between the analytical prediction and numerical simulation of the GP equation is also presented. Since there is no stable localized solution (like dark soliton in $1 \mathrm{D}$ repulsive case) of the GP equation in higher dimensional settings, creation of density disturbance in this case also can be done by illuminating the homogeneous mixture BEC by tightly focused laser beam designed to change the intra- or interspecies interaction constant via optically induced Feshbach 

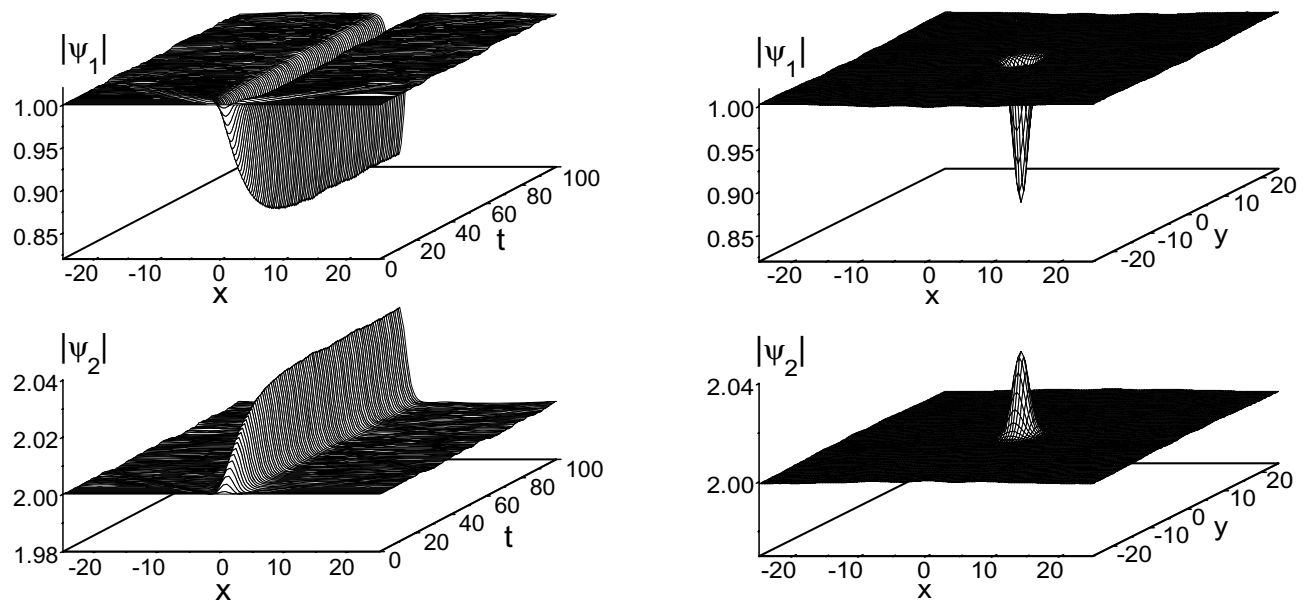

Figure 6. Formation of a dark-bright density defect in a 2D homogeneous mixture of two repulsive BECs when the intra-species repulsion is locally increased in one of the components according to law $g_{11}(x, y, t)=g_{11}^{0}+2 G(x, y) \tanh \left(5 t / t_{\text {end }}\right)$, where

$G(x, y)=1 /\left(\pi a^{2}\right) \exp \left(-\left(x^{2}+y^{2}\right) / a^{2}\right)$ is a Gaussian perturbation of the intra-species interaction constant. The initial state and parameters are taken as $\psi_{1}(x, y)=1, \psi_{2}(x, y)=2, g_{11}^{0}=1, g_{22}=1, g_{12}=0.5, a=1, t_{\text {end }}=100$. Left panel: the time evolution of the BEC wave amplitudes $\psi_{1,2}(x, y)$ according to the coupled GP equation shown at the $y=0$ cross section during adiabatic change of the coefficient $g_{11}$. Right panel: a dark-bright localized state emerged in a mixture of two repulsive BECs with finite backgrounds in all repulsive case at $t=t_{\text {end }}$.
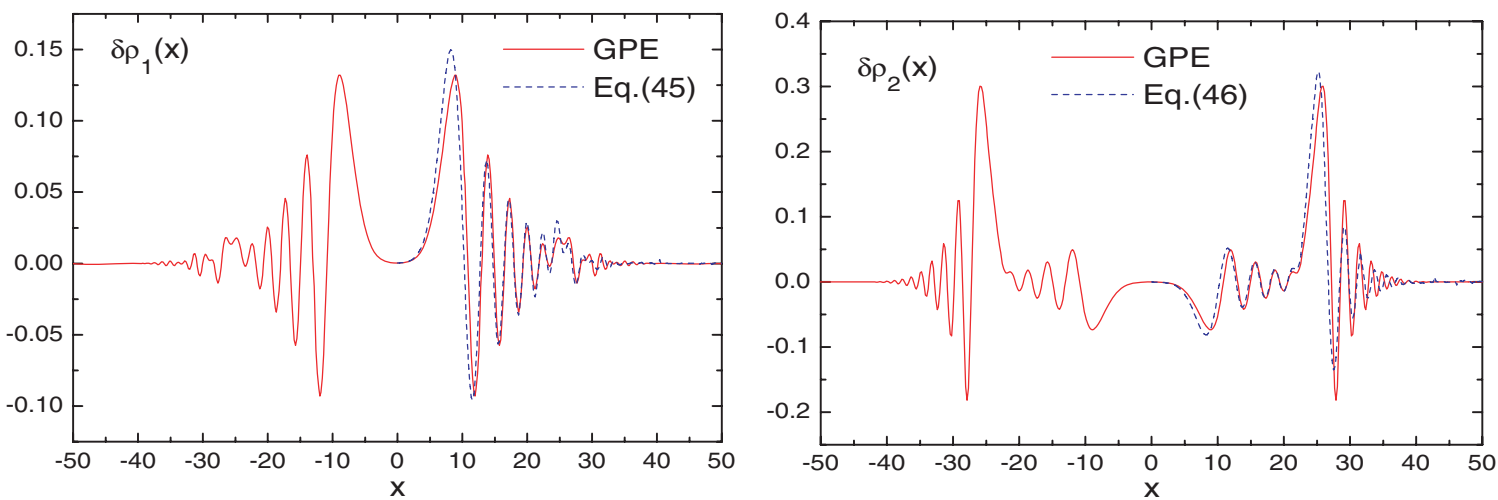

Figure 7. Density modulations at $t=8$ according to equations (45)-(46) and numerical solution of the coupled GP equation. Parameters: $\rho_{10}=1, \rho_{20}=9, a=1, g_{11}=1, g_{22}=1, g_{12}=0.5$.
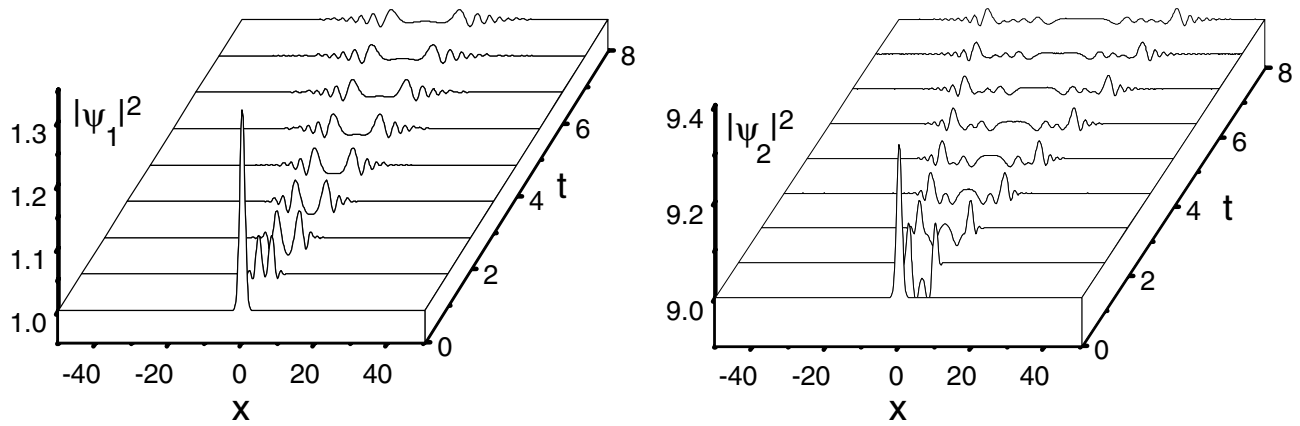

Figure 8. Evolution of waves in a 2D mixture BEC emerged from the decay of a Gaussian density disturbance with $a=1$ in both components shown at the $y=0$ cross section according to numerical solution of the coupled GP equations. Background wave amplitudes of components and nonlinear coefficients are equal to $\psi_{1}(x, y)=1, \psi_{2}(x, y)=3, g_{11}=1, g_{22}=1, g_{12}=0.5$. Due to different sound velocities in the components radially outward travelling waves quickly separate.

resonance. In figures $8-10$ the generation, further evolution and pattern of linear waves at particular time are depicted for the $2 \mathrm{D}$ coupled repulsive BECs. As can be seen from figures 4, 7 and 10, the agreement between analytical predictions and numerical solution of the GP equation is quite good. 

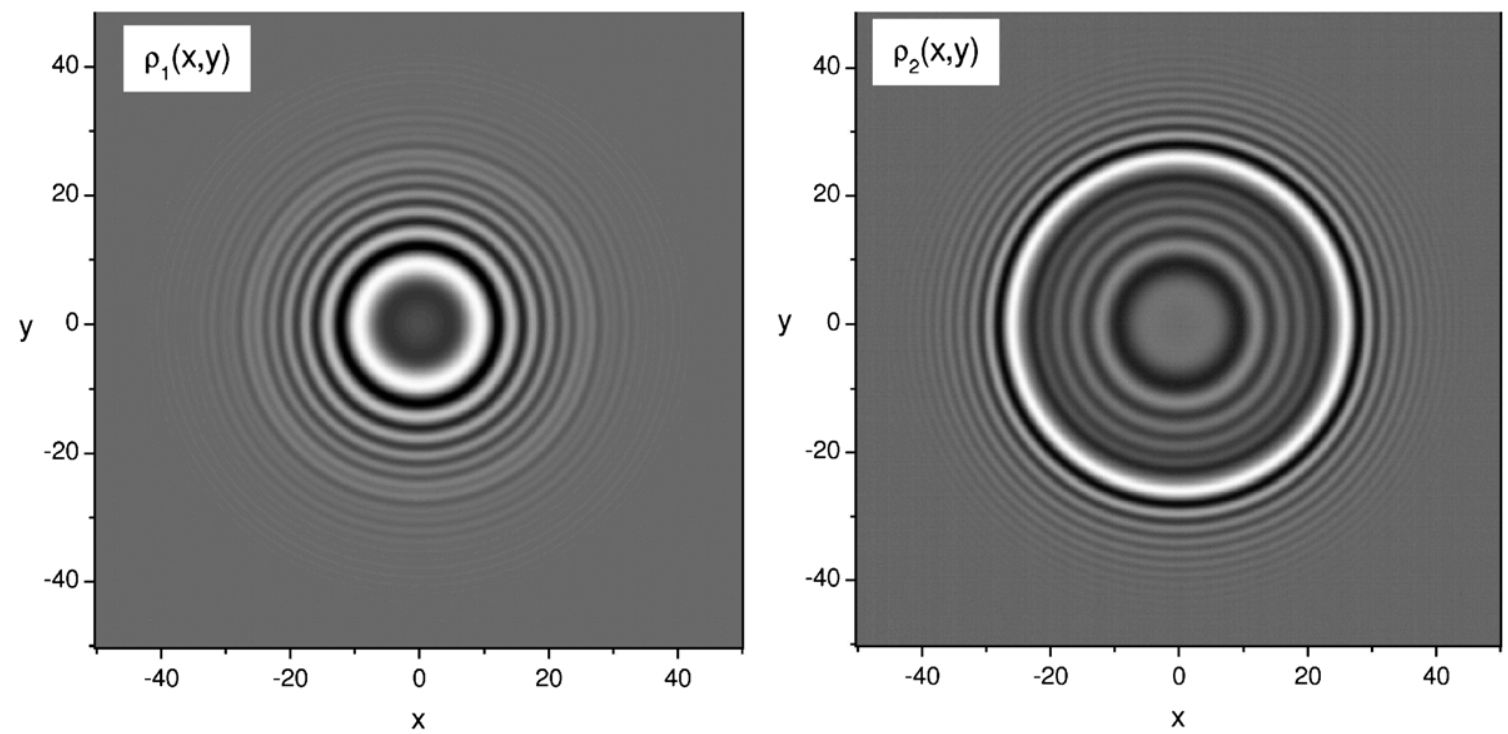

Figure 9. Density plots of two components corresponding to the last time section at $t=8$ of the previous figure 8 . In white areas the density is higher. Separation of waves in the two components due to different sound velocities is clearly seen.
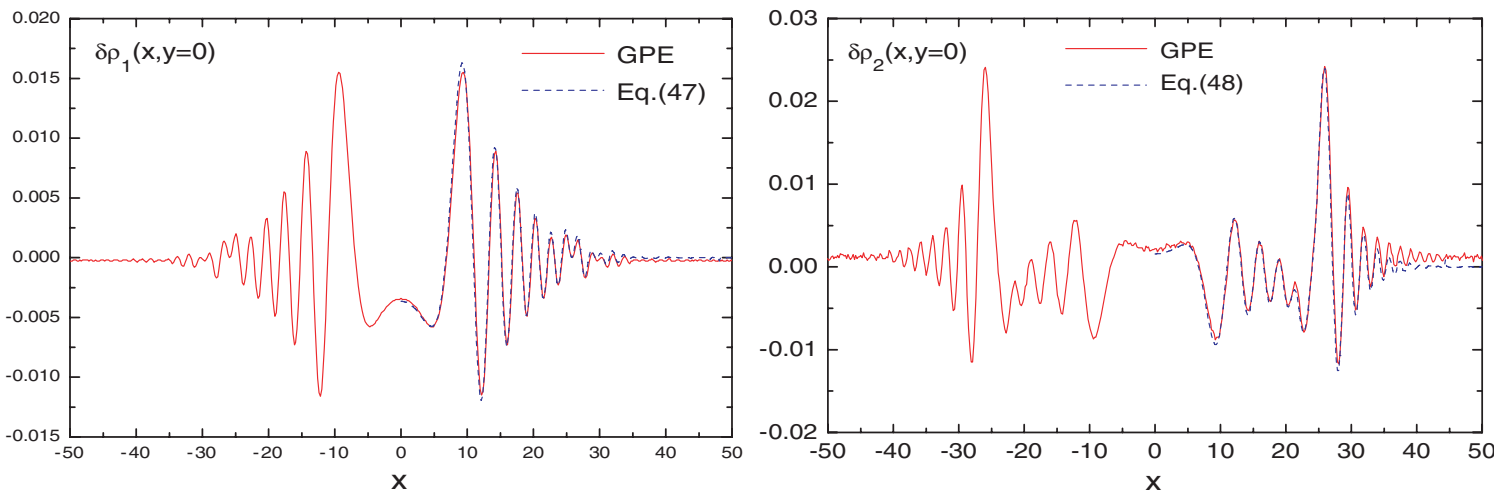

Figure 10. Comparison of density distributions provided by equations (47) and (48) with numerical solution of the coupled GP equation for the same parameters as in figure 8 for $t=8$. Relative positions of the wave maximums in the second and first modes $26 / 9 \simeq 3$ well match the ratio of corresponding sound velocities $c_{+} / c_{-}$which have the same order of magnitude as group velocities of our wave packets built of harmonics with wavelengths about healing length.

\section{Conclusions}

We have investigated sound waves in two-component BECs and proposed a new method of wave generation which is based on a fast change of the inter-species interaction constant. To illustrate the idea, we have performed numerical simulations of the creation of a density defect (hump or hole) on the background condensate and its evolution into matter wave-packets after the fast change of the interspecies interaction constant. This method of wave generation was used to investigate both numerically and analytically sound waves arising from the release of a drop-like condensate immersed into a second large repulsive condensate and from homogeneous mixture of two repulsive BECs both with finite backgrounds. We demonstrated that propagation of linear waves created by this approach is well described by the Gross-Pitaevskii equation. Explicit analytical formulae were obtained for the spacetime evolution of density waves in coupled BECs, starting from the linearized GP equations.
The comparison of the analytical results with direct numerical simulations of the coupled GP equations showed an excellent agreement in spite of nonlinearity of the GP equation. Our study also sheds light on the difference between linear wave packets and dispersive shocks which is sometimes overlooked. In particular, it is clear from our analytical results that the linear wave packets propagate with the velocity greater, due to dispersion effects, than the sound velocity defined for the infinite wavelength limit. On the opposite, the dispersive shocks which represent the soliton trains can propagate with velocities less than the sound velocity. This sharp distinction between apparently similar effects permits one easily distinguish them.

Although in this paper we have restricted our considerations to the instant weak density disturbances (e.g. amplitude of the disturbance is small compared to the amplitude of the background condensate), the proposed method can be also used for investigation of ship waves 
created by a permanent moving source of a disturbance and this problem will be discussed elsewhere.

\section{Acknowledgments}

BBB acknowledges partial support from the Fund for Fundamental Research of the Uzbek Academy of Sciences under Grant No 10-08. AMK wishes to thank the Department of Physics 'E R Caianiello' of the University of Salerno, where part of this work was done, for the hospitality received and for financial support.

\section{References}

[1] Carretero-Gonzalez R, Frantzeskakis D J and Kevrekidis P G 2008 Nonlinearity 21 R139

[2] Gurevich A V and Krylov A L 1987 Zh. Eksp. Teor. Fiz. 92 1684

[3] El G A, Geogjaev V V, Gurevich A V and Krylov A L 1995 Physica D 87186

[4] Kamchatnov A M, Kraenkel R A and Umarov B A 2002 Phys. Rev. E 66036609

[5] Brazhnyi V A and Kamchatnov A M 2003 Phys. Rev. A 68043614

[6] Kamchatnov A M, Gammal A and Kraenkel R A 2004 Phys. Rev. A 69063605

[7] Hoefer M A, Ablowitz M J, Coddington I, Cornell E A, Engels P and Schweikhard V 2006 Phys. Rev. A 74023623

[8] Konotop V V and Salerno M 1997 Phys. Rev. E 563611

[9] Abdullaev F Kh, Baizakov B B, Darmanyan S A, Konotop V V and Salerno M 2001 Phys. Rev. A 64043606

[10] Menotti C, Krämer M, Smerzi A, Pitaevskii L and Stringari S 2004 Phys. Rev. A 70023609

[11] Simula T P, Engels P, Coddington I, Schweikhard V, Cornell E A and Ballagh R J 2005 Phys. Rev. Lett. 94080404

[12] Myatt C J, Burt E A, Ghrist R W, Cornell E A and Wieman C E 1997 Phys. Rev. Lett. 78586

Hall D S, Matthews M R, Ensher J R, Wieman C E and Cornell E A 1998 Phys. Rev. Lett. 811539

Stamper-Kurn D M, Andrews M R, Chikkatur A P, Inouye S, Miesner H J, Stenger J and Ketterle W 1998 Phys. Rev. Lett. 802027

Stenger J, Inouye S, Stamper-Kurn D M, Miesner H J, Chikkatur A P and Ketterle W 1998 Nature (London) 396345

Miesner H J, Stamper-Kurn D M, Stenger J, Inouye S, Chikkatur A P and Ketterle W 1999 Phys. Rev. Lett. 822228

[13] Modugno G, Modugno M, Riboli F, Roati G and Inguscio M 2002 Phys. Rev. Lett. 89190404

Catani J, De Sarlo L, Barontini G, Minardi F and Inguscio M 2008 Phys. Rev. A 77011603
Thalhammer G, Barontini G, de Sarlo L, Catani J, Minardi F and Inguscio M 2008 Phys. Rev. Lett. 100210402

[14] Kostov N A, Enolskii V Z, Gerdjikov V S, Konotop V V and Salerno M 2004 Phys. Rev. E 70056617

Cruz H A, Brazhnyi V A, Konotop V V, Alfimov G L and Salerno M 2007 Phys. Rev. A 76013603

Abdullaev F Kh, Gammal A, Salerno M and Tomio L 2008 Phys. Rev. A 77023615

[15] Brazhnyi V A and Konotop V A 2005 Phys. Rev. A 72026616

[16] Bruderer M, Bao W and Jaksch D 2008 Europhys. Lett. 8230004

[17] Carusotto I, Hu S X, Collins L A and Smerzi A 2006 Phys. Rev. Lett. 97260403

[18] Gladush Yu G, El G A, Gammal A and Kamchatnov A M 2007 Phys. Rev. A 75033619

[19] Gladush Yu G and Kamchatnov A M 2007 JETP 105520

[20] Gladush Yu G, Smirnov L A and Kamchatnov A M 2008 J. Phys. B: At. Mol. Opt. Phys. 41165301

[21] Goldstein E V and Meystre P 1997 Phys. Rev. A 552935

[22] Andrews M R, Kurn D M, Miesner H-J, Durfee D S, Townsend C G, Innouye S and Ketterle W 1997 Phys. Rev. Lett. 79553

[23] Liang Z X, Dong Xi, Zhang Z D and Wu B 2008 Phys. Rev. A 78023622

[24] Susanto H, Kevrekidis P G, Carretero-Gonzalez R, Malomed B A, Frantzeskakis D J and Bishop A R 2007 Phys. Rev. A 75055601

[25] Dutton Z, Budde M, Slowe Ch and Hau L V 2001 Science 293663

[26] Papp S B and Wieman C E 2006 Phys. Rev. Lett. 97180404 Papp S B, Pino J M and Wieman C E 2008 Phys. Rev. Lett. 101040402

Li Z, Singh S, Tscherbul T V and Madison K W 2008 Phys. Rev. A 78022710

[27] Press W H, Teukolsky S A, Vetterling W T and Flannery B P 1996 Numerical Recipes. The Art of Scientific Computing (Cambridge: Cambridge University Press)

[28] Agrawal G P 1995 Nonlinear Fiber Optics 2nd ed (New York Academic)

[29] Minguzzi A, Succi S, Toschi F, Tosi M P and Vignolo P 2004 Phys. Rep. 395223

[30] Bao W 2004 Multiscale Model. Simul. 2210

[31] Chiofalo M L, Succi S and Tosi M P 2000 Phys. Rev. E 627438

[32] Fedichev P O, Kagan Yu, Shlyapnikov G V and Walraven J T M 1996 Phys. Rev. Lett. 772913

[33] Theis M, Thalhammer G, Winkler K, Hellwig K, Ruff G, Grimm R and Denschlag J H 2004 Phys. Rev. Lett. 93123001

[34] Tiesinga E, Verhaar B J and Stoof H T C 1993 Phys. Rev. A 474114

[35] Busch Th and Anglin J R 2001 Phys. Rev. Lett. 87010401

[36] Öhberg P and Santos L 2001 Phys. Rev. Lett. 862918

[37] Kevrekidis P G, Nistazakis H E, Frantzeskakis D J, Malomed B A and Carretero-González R 2004 Eur. Phys. J. D 28181

[38] Becker C, Stellmer S, Soltan-Panahi P, Dorscher S, Baumert M, Richter E M, Kronjager J, Bongs K and Sengstock K 2008 Nature Phys. 4496 